\title{
Revision Of A Standard For The Test Of A Toilet-Incorporated Medical Electric Bed
}

\author{
Ho Jun Yeom ${ }^{1,}$ Seokmin Lee ${ }^{2}$, Kiwon Choi ${ }^{3}$, In-Ho Hwang ${ }^{4,}$ Sangsoo Park*5 \\ 1,5* Department of Biomedical Engineering, Eulji Univ.,Seongnam, Republic of Korea \\ 2,3,4 Korea Orthopaedic and Rehabilitation Center, Incheon, Republic of Korea \\ hjyeom@eulji.ac.kr ${ }^{1}$, retrometro26@kcomwel.or.kr², cgw3321@kcomwel.or.kr ${ }^{3}$, ihhwang@kcomwel.or.kr ${ }^{4}$, \\ spark@eulji.ac.kr ${ }^{5}$
}

Article History: Received: 11 January 2021; Accepted: 27 February 2021; Published online: 5 April 2021

\begin{abstract}
We developed a toilet-incorporated electric medical bed for bedridden patients, in which a toilet basin, or a commode, is incorporated in the pelvic plate of an electric medical bed. A replaceable toilet seat is located in the mattress of the bed, and toilet basin was tightly attached on the pelvic plate the bed. The excretion slides through a storage tube that angles toward the side of the bed and is automatically collected in a disposable plastic bag. We, however, encountered a problem with testing this newly developed medical bed with the relevant international standard IEC 60601-2-52:2009, or with the corresponding Korean standard KS P 0388:2012-Electrically operated adjustable bed for home care. There was no clear indication in the scopes of the above standards as to whether this standard testing method is applicable to an electric medical bed embedded with a commode. We revised the Korean standard KS P 0388:2012 to accommodate the toilet-incorporated medical bed in the scope of the standard and successfully performed the mechanical tests including the durability, impact, and deformation test of the bed, proving that the newly developed toilet-incorporated medical bed is mechanically strong and durable enough to pass the standard tests.
\end{abstract}

Keywords: Toilet-incorporated, electric medical bed, mechanical test, toileting device, standard revis

\section{Introduction}

Falls by the elderly are caused by physical and mental decline as a result of aging, and about half of them are associated with toileting [1-3]. To minimize the risk of toileting-related fall, there have been many ideas for installing a toileting device in or near a medical bed, but these ideas have not been materialized enough to be useful in practice and accepted by the public [4-6].

The idea of installing a toileting aid in a medical bed was reported first in 1998 by Fujimoto et al [4]. Their toileting aid, an automatic excretion handling system, comprised of an excretion collection cup, a fluid waste storage container, a suction pump, and a controller of the system. The excretion collection cup was placed in between the thighs of the patient and a diaper was wrapped around the patient's pelvis for maintaining the position of the excretion collection cup. The excrement collected in the excretion collection cup was drawn to a fluid waste storage container inside a main body that is placed near the bed through a connecting tube. The main body also houses the suction pump and the controller of the system. The suction pump is turned on upon detection of urine or feces in the excretion collection cup by the sensors imbedded in the cup. The basic structure of the bed was not changed as the excretion collection cup is on or inside the mattress of the medical bed. The system has been commercialized in Japan and Korea with limited acceptance by the patients and carers, as it has some inherent disadvantages [5-6], such as inconvenience for the patient, as the patient has a difficulty in turning his/her body with the bulky excrement collection cup in between the thighs and cleaning difficulty as the channel between the collection cup and the storage container is long and narrow.

These problems could be overcome if an excrement collection device were placed under the mattress below the patient buttock, and if the channel between the collection part and the storage part were wide enough, and thus developed a toilet-incorporated electric medical bed that suffices both the conditions and previously reported a case study of the new system [7-12]. This new excrement disposal system should however solve the safety issues related with incorporating a toilet in an electric medical bed as the commode incorporation modifies the plate of the bed significantly. We deal with the safety issues related with the mechanical stability and durability in relation to the incorporation of the toilet in an electric medical bed in this paper

2. Toilet-incorporated electric medical bedFigure 1 illustrates the toilet-incorporated electric medical bed we developed. The bed is composed of five plates: head, back, pelvis, upper leg, and lower leg plates. Head board, foot board, side rails, tray table is similar to other electric beds, but the core difference lies in the presence of a shower head on the head board and a toilet attached to the pelvis plate. The part of the mattress where the pelvis plate is located is cut to the shape of a toilet seat, and is normally covered by a matching mattress when the toilet is not in use. For toilet usage, the cover is removed, and a polyurethane toilet seat is fitted into the position. 
Figure 2 shows lower portion of the toilet, a stainless steel toilet basin (A) is attached to the pelvis plate by six bolts and nuts. Fixed to the pelvis plate above, the toilet basin is connected to a storage tube (B) angled at the lower part and exposed at its terminal toward the side under the bed. The storage tube is to store the fluid waste temporarily before disposal by the patient carer. A disposal unit, $\mathrm{C}$ is attached with a bracket to the angled storage tube, inside which a polyethylene film bag $(18 \mathrm{~cm}$ in diameter and $15 \mathrm{~m}$ in length) is rolled around. When the lid (D) covering the storage tube is opened, the fluid waste in the tube flows down into the polyethylene bag by the force of gravity. The bag with the fluid waste is then pulled and clipped, to dispose of the content in the toilet in the bathroom. The remaining bag is tied at its end, and the lid to the storage tube is closed, ready for use in the next toileting event.

\section{Problems in Mechanical Test}

The international standard applicable to the electric medical bed for patient use is IEC 60601-2-52:2009 [8]. In Korea, the standard for electric medical beds is included in medical equipment standards, Notification 201872 by the Ministry of Food and Drug Safety [9].

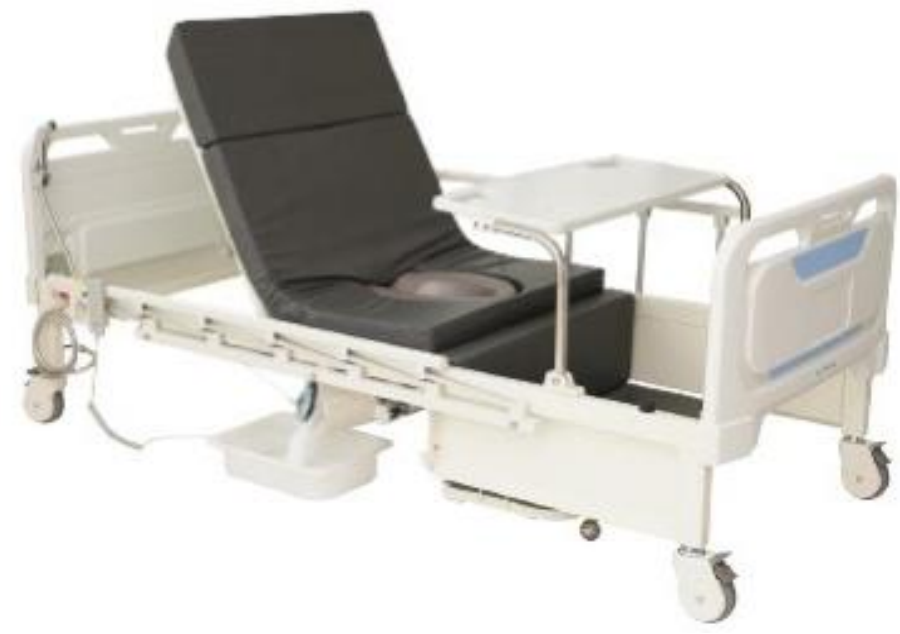

Figure 1. Toilet-incorporated electric medical bed

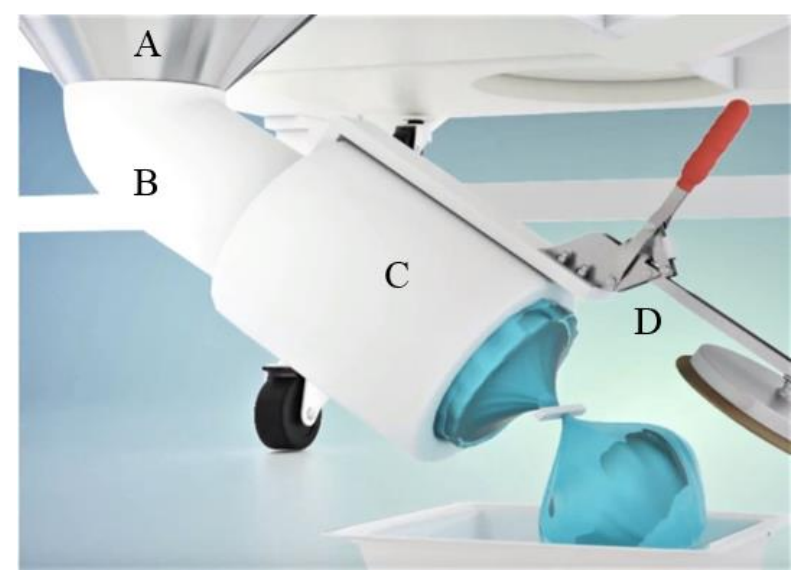

Figure 2. Excrement collection system under the bed

In addition, KS P 0388:2012--Electrically operated adjustable bed for home care, also stipulates the test standards for electric medical bed for home use [10]. Because this product is designed mainly for elderly care at home, we planned to comply with the KS P 0388:2012 standard set out for electric beds for home care. However, we encountered the following issues in applying KS P 0388:2012 to this toilet-incorporated medical bed.

1) There is no clear regulation that indicates whether KS P 0388:2012 is applicable to an electric bed embedded with a commode. According to KS P 0388:2012, only the foot board, head plate, mattress-supporting plate, divided side rail, switch, locking latch on the side rail, lower frame, and side frame are defined as components of an electric medical bed. The meal tray, which is commonly included for use, is not defined as a component of a medical bed. It was therefore questionable whether a product with a commode incorporated to the pelvis plate can be tested pursuant to the KS P 0388:2012. It was also not clear whether this medical bed could be tested by the method stipulated in IEC 60601-2-52:2009, as the standard defines only lifting pole, 
intravenous (IV) pole, side rails, fracture frames as an accessory to a medical bed. In other words, it was not clear whether the electric medical bed incorporated with a commode is within the scope of KS P 0388:2012 and/or IEC 60601-2-52:2009.

2) Even if KS P 0388:2012 is applicable to an electric bed that integrated with a commode, it was unclear whether the existing mechanical safety test standards could be employed and the toilet-bed could pass the test, as the pelvis plate was significantly modified to accommodate the basin of the toilet.

\section{Resolution of the Problems}

To resolve these problems, the Korea Rehabilitation Engineering Research Center (KOREC), the standard cooperation agency of the Korean Agency for Technology and Standards (KATS), summoned an expert committee. Based on the feedback from the meeting, the problems were resolved in the following manner.

1) KS P 0388:2012 was revised by adding a paragraph in the introduction part, to include this toiletincorporated bed in the scope of the standard [11]. The wording added to the introduction part is as follows: Owing to recent technological advancements, supplementary components are added to products for enhanced convenience for patients. These components include meal trays for patients, poles to support patients to stand and exercise, shower apparatus to wash patients' faces and hair, and a toilet for excrement disposal. Because these components should not affect the safety of the bed, available components must be fitted to the bed for testing according to the standards. However, the performance and safety of each component shall be excluded from the standards".

Pursuant to the new wording added to the introduction of the standard, KS P 0388:2019 is now applicable to electric beds enhanced with new components for greater performance [11].

2) After revision of the Korean standard, the mechanical test in IEC 60601-1-52 and KS P 0388:2019 was applied to the toilet bed: durability, impact, and deformation tests were conducted on the locations shown in Figure 3.

A. Durability test: A test mattress was laid on the flat bed. A 75-kg load pad (750 N) was used to apply pressure to Position A 10,000 times. The load pad was a hard, round object measuring $355 \mathrm{~mm} \pm 5 \mathrm{~mm}$ in diameter. A spherical convex with a curvature radius of $800 \mathrm{~mm}$ was applied to it, with a radius of $20 \mathrm{~mm}$ to the front. After the load test, the test mattress was removed and it was confirmed that the tested product functioned normally without any evidence of structural deterioration

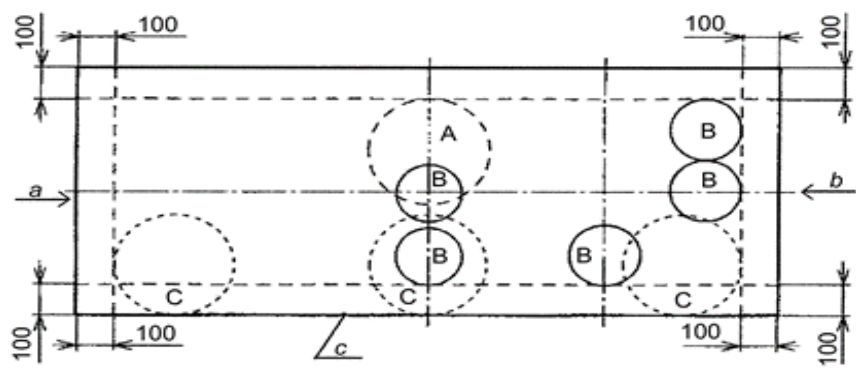

Figure 3. Loading positions for the test of electric medical bed: A for the durability test, B for the impact resistance test, and $\mathrm{C}$ for the deformation test.

I.

B. Impact resistance test: The bed was positioned horizontally, and a test mattress was placed on the bed. The impact points at four different positions were marked (Point B). Impacts were applied 20 times for each mark using an impactor, a rigid circular object with $200 \mathrm{~mm}$ in diameter and with an assembled mass of $17.0 \mathrm{~kg}$. The test mattress was removed, and it was confirmed that the bed functioned normally and all operations of the bed were possible without any difficulty.

C. Deformation test: The bed was positioned horizontally, and a test mattress was placed on the bed. Three 75$\mathrm{kg}$ load pads were applied each time to Positions C. The extent of deformation was measured before, during, and after load application, to identify any abnormalities with components. The deformation was much less than the criteria; it was less than $2.0 \mathrm{~mm}$ during the load application and was less than $1 \mathrm{~mm}$ after removal of the load pads

\section{Discussion}

The pelvis plate of this toilet-incorporating bed was cut to accommodate the oval-shape toilet basin with a long diameter of $290 \mathrm{~mm}$ and a short diameter of $210 \mathrm{~mm}$. There were questions regarding whether the mechanical testing method of KS P 0388:2012 could be applied to this product, as the opening of the toilet basin overlapped with the positions of the load pads used in the durability, deformation, and impact tests of KS P 
0388:2012. This concern was easily resolved since the durability, deformation, and impact tests was performed with a mattress on the system, as required by KS P 0388:2012. The existence of the vacant hole of the toilet basin did not interfere with the mechanical tests, since the load was not applied directly on the bed plate but on the mattress above the bed. This toilet-incorporated electric bed passed all three mechanical tests without any issue. If there were a test category that required mechanical test of the bed without the mattress, it would not be feasible to apply KS P 0388:2012 to the mechanical test on the bed with the commode attached in the pelvic plate of the bed.

\section{Conclusions}

A toilet-incorporated medical electric bed was tested with a Korean Standard KS P 0388:2019, and was proved mechanically strong and durable enough. The test, however, required a revision of the standard, since this type of electric medical bed had not been included in the scope of the previous version of the standard. It is strongly recommended that the corresponding international standard IEC 60601-2-52:2009 be revised to accommodate new multifunctional medical nursing beds.

\section{References}

1. Decalf, V., Bower, W., Rose, G., Petrovic, M., Pieters, R., Eeckloo, K. and Everaert, K., 2019. Prevalence and characteristics of incident falls related to nocturnal toileting in hospitalized patients. Acta Clinica Belgica, pp.1-6.

2. Sakushima, K., Yamazaki, S., Fukuma, S., Hayashino, Y., Yabe, I., Fukuhara, S. and Sasaki, H., 2016. Influence of urinary urgency and other urinary disturbances on falls in Parkinson's disease. Journal of the Neurological Sciences, 360, pp.153-157.

3. Mahmoodabad, S.S.M., Zareipour, M., Askarishahi, M. and Beigomi, A., 2018. Effect of the Living Environment on falls among the Elderly in Urmia. Open access Macedonian Journal of Medical Sciences, 6(11), p.2233.

4. Fujimoto, T., Hashimoto, T., Sakaki, H., Higashi, Y., Tamura, T., \& Tsuji, T. (1998) Automated handling system for excretion. In Proceedings of the 20th Annual International Conference of the IEEE Engineering in Medicine and Biology Society. Vol. 20 Biomedical Engineering Towards the Year 2000 and Beyond (Cat. No. 98CH36286) (Vol. 4, pp. 1973-1976). IEEE.

5. Koh, E. J., \& Park, S. S. (2015). Technical and Commercialization Status of Urine and Feces Disposal Systems. Journal of Rehabilitation Welfare Engineering \& Assistive Technology, 9(2), 169-175

6. Park, S., Choi, H. W.., and Ryu, D.S. (2016). A feces collection bag for bedridden patients with fecal incontinence. The 2nd International Integrated(Web \& Offline) Conference \& Concert on Convergence, St. Petersburg, Russia.

7. Yeom, H., Park, C. H., \& Park, S. (2019). Medical bed with integrated toilet: design considerations and utilization by a bedridden patient. Health and Technology, 9(5), 903-907.

8. International Electrotechnical Commission. IEC 60601-2-52:2009 Medical electrical equipment -- Part 2-52: Particular requirements for the basic safety and essential performance of medical beds.

9. Korean Ministry of Food and Drug Safety. Medical Device Standard Specifications, Korea Department of Food and Drug Safety Notification 2018-72.

10. Korean Standard Agency.KS P 0388:2012 Electrically operated adjustable bed for home care.

11. Korean Standard Agency. KS P 0388:2019 Electrically operated adjustable bed for home care.

12. AY, T. S., \& Zeynep, Ö. N. C. Ü. (2019). An Analysis of the Documentary Entitled Talisman of the City as a Teaching Tool in the Values Education. Review of International Geographical Education Online, 9(3), 639-662. 